\title{
Hermansky-Pudlak syndrome in pregnancy: A case report
}

\section{Lydia Yusuf', Srivasavi Dukka' and Etienne Ciantar ${ }^{2}$}

\begin{abstract}
Hermansky-Pudlak syndrome is a rare autosomal recessive disorder estimated to affect I in 500,000 to I,000,000 individuals worldwide. Clinically, it presents as oculocutaneous albinism combined with bleeding diathesis. This is due to the absence of dense bodies in platelets causing a delayed secondary response resulting in prolonged bleeding time despite normal platelet count and coagulation factors. This has consequences for major bleeding, the risk of which is high at delivery. In the longer term, the condition is also associated with the development of pulmonary fibrosis, inflammatory bowel disorders caused by granulomatous colitis and renal failure. We present a case of a patient with Hermansky-Pudlak syndrome diagnosed and managed through her second pregnancy by the Obstetric Haematology team in a Tertiary Unit. During her previous pregnancy at a District Hospital, they had not been aware of the diagnosis; she then had a forceps delivery and her haemoglobin dropped to $69 \mathrm{~g} / \mathrm{L}$. During this pregnancy, she was managed in a multidisciplinary setting involving obstetrics, haematology, anaesthesia and neonatology. She was induced at 39 weeks and had human leukocyte antigen-matched platelets prepared for her delivery. Her platelet count was normal throughout the pregnancy. She had a normal vaginal delivery under platelet cover and also received tranexamic acid at the time of cord clamping. Her blood loss was moderate and her haemoglobin dropped from II 5 to $97 \mathrm{~g} / \mathrm{L}$. She recovered well. We discuss the diagnosis, pathology and management of this very rare condition in pregnancy and thereafter.
\end{abstract}

\section{Keywords}

Haematology, high-risk pregnancy, physiology, transfusion, platelets

Date received: 30 October 2015; accepted: 7 July 2016

\section{Introduction}

Hermansky-Pudlak syndrome (HPS) is a clinical diagnosis established by findings of oculocutaneous albinism in combination with a bleeding disorder of variable severity due to platelet dysfunction.

Hermansky and Pudlak originally described this diagnosis in $1959^{2}$ following the assessment of two unrelated individuals, both had albinism with a bleeding tendency and pigmented reticular cells in the bone marrow as well as in lymph node and liver biopsies. ${ }^{2}$ It was identified as different from Chediak-Higashi syndrome, a rare condition of oculocutaneous albinism, bleeding diathesis and recurrent infection, because no neutrophil inclusions are found in HPS and no pigmented macrophages are found in Chediak-Higashi syndrome ${ }^{3}$ as was discovered in this case. In addition, HPS is not associated with immunosuppression.

HPS is a rare autosomal recessive disorder and is estimated to affect 1 in 500,000 to $1,000,000$ individuals worldwide. ${ }^{4}$ This incidence is markedly higher in Puerto Rico where it is said to be as high as 1 in $800 .{ }^{5}$

\section{Case}

A 31-year-old Caucasian female in her second pregnancy presented to the Obstetric Haematology clinic. The patient was noted to have oculocutaneous albinism, marked nystagmus and had a history of bleeding diathesis.

A detailed history revealed that the patient had always bruised easily and had bled significantly as a child following a dental extraction although she denied having heavy menstrual bleeding. She had always been noted to have albinism since birth. Genetic consultation during her first pregnancy had proposed the diagnosis of Chediak-Higashi syndrome, a condition associated with oculocutaneous albinism combined with platelet disorder, recurrent infections and a bleeding diathesis. Although she demonstrated evidence of a platelet release defect, she had no history of recurrent infections and blood film showed no neutrophil inclusions, which was therefore more in keeping with a diagnosis of HPS.
Her previous delivery was at a District Hospital where she was induced at 37 weeks for pre-eclampsia. She had been treated with anti-hypertensives and had proteinuria from 36 weeks gestation. There were platelets on standby at the time of delivery, due to the presumed diagnosis of Chediak-Higashi syndrome, but these were not given. She had a forceps delivery but was unsure of the indication. Her estimated blood loss was not known; however, her haemoglobin dropped to $69 \mathrm{~g} / \mathrm{L}$ postnatally and she was given oral iron tablets. The child had no features of albinism or of easy bruising and did not seem to exhibit any features of the genetically inherited syndrome.

Antenatal care in the index pregnancy was carried out in a joint Obstetric Haematology clinic in a Tertiary Unit involving obstetrics, haematology, anaesthesia and neonatology. It was important to check human leukocyte antigen (HLA) type and HLA antibody status in the antenatal period as the patient would require HLA-matched platelets prophylactically during labour. This was done at 28 weeks gestation and there were no HLA or human platelet antigen antibodies detected in this patient. She had fortnightly blood pressure and urine checks from 20 weeks in view of her history of pre-eclampsia in her previous pregnancy.

\section{Delivery}

The patient was admitted for induction of labour at $39+6$ weeks. She was found to be suitable for artificial rupture of membranes on admission. She was promptly transferred to Delivery Suite.

'Leeds Teaching Hospitals, NHS Trust, Leeds, UK

'Division of Women's and Children's Health, Leeds Teaching Hospitals, NHS Trust, Leeds, UK

\section{Corresponding author:}

Lydia Yusuf, Leeds Teaching Hospitals, NHS Trust, Great George Street, Leeds LSI 3EX, UK.

Email: lydiayusuf@doctors.org.uk 
The HLA-matched platelet transfusion commenced after rupture of membranes at 23:00. The delivery plan stipulated that the patient was to have a second bag of HLA-matched platelets during the first stage of labour and then a third bag during second stage. Tranexamic acid was also recommended at delivery. Labour progressed spontaneously and she was contracting well by $23: 55$. Her labour rapidly culminated in a spontaneous vaginal delivery at 02:23. Estimated blood loss was $800 \mathrm{~mL}$.

Only two units of HLA-matched platelets were completed as labour progressed quickly. She was given intramuscular syntocinon and an intravenous syntocinon infusion postnatally. The third stage was managed actively and she received tranexamic acid at the time of cord clamping. Her haemoglobin dropped from 115 to $9 \mathrm{~g} / \mathrm{L}$. Her platelet count was normal throughout pregnancy, as is typical of the condition.

The patient was followed up postnatally when she was found to be doing well with normal lochia and no symptoms of anaemia. She will be followed up under the care of haematology to monitor for any complications associated with HPS. She will have regular pulmonary function tests and chest X-rays and be advised to report any abnormal bowel symptoms.

\section{Discussion}

The identification of HPS begins clinically with a diagnosis of oculocutaneous albinism, which is established by finding hypopigmentation of the skin and hair on physical examination. ${ }^{6}$ It is associated with characteristic ocular findings of nystagmus, reduced iris pigment with iris transillumination, decreased retinal pigment on fundoscopic examination and foveal hypoplasia associated with significant reduction in visual acuity. ${ }^{6}$

The bleeding diathesis can result in easy bruising, frequent epistaxis, gingival bleeding, postpartum haemorrhage, colonic bleeding and prolonged bleeding with menses or after dental procedures. ${ }^{6}$

Investigations for HPS include whole mount electron microscopy. The characteristic finding is absence of dense platelet bodies. Dense bodies, which contain adenosine triphosphate, adenosine diphosphate, serotonin, phosphate and calcium, act to attract other platelets by releasing their contents. ${ }^{7}$ This constitutes the secondary aggregation response on platelet aggregation studies. In individuals with HPS, this cannot occur due to the absence of dense bodies to most agonists.? Therefore, as the secondary response of platelets is impaired, bleeding time may be prolonged although the platelet count and coagulation factors will be normal. ${ }^{7}$ Platelet nucleotide studies demonstrate the absence of platelet $\delta$-granule secretion whereas $\alpha$-granule secretion is normal. Confirmation is by molecular genetic analysis and allows classification into a particular HPS subtype (HPS1-8) which is important for therapy and prognosis. ${ }^{8}$

Long-term implications of HPS are due to associated complications such as pulmonary fibrosis, inflammatory bowel problems caused by granulomatous colitis and renal failure. ${ }^{7,9}$ In HPS, pulmonary fibrosis is caused by deposition of ceroid-lipofuscin, a lipid protein complex, within the alveolar septae and causes symptoms which commonly appear during the patient's early 30 s and rapidly worsen. ${ }^{7,10}$ Nine different types of HPS have been described. Their signs and symptoms and the underlying genetic cause differentiate them. Types 1 and 4 are the most severe where types 3,5 and 6 are the mildest forms. Pulmonary fibrosis is associated with types 1,2 and $4 .{ }^{1,7}$

Initial management begins with correction of refractive errors and use of low vision aids. The use of thrombin-soaked gelfoam for skin wounds with prolonged bleeding is described. ${ }^{7}$ Antifibrinolytic agents like tranexamic acid may be used for minor procedures and bleeds. If platelet aggregation improves with 1-desamino-8-D-arginine vasopres$\sin$, it can be given prior to invasive procedures ${ }^{11}$ and ultimately platelet or red cell transfusions may be required for surgery or prolonged major bleeding. ${ }^{7}$

In the event of severe haemorrhage, platelet transfusion would be effective in controlling bleeding but may lead to the development of alloimmunisation. ${ }^{12}$ Indeed, because of the risk of alloimmunisation in patients with thrombocytopathies, platelet transfusions should be used only for the treatment of severe bleeding. The HLA antibody mediated destruction of the transfused platelets can induce refractoriness to platelet transfusions. ${ }^{13}$ Care should be taken to limit the risk of alloimmunisation by limiting exposure and using pre-stored and leucodepleted platelet concentrates or HLA-matched donors for transfusion. ${ }^{12}$

Alloimmunisation can also cause posttransfusion purpura, a rare immune phenomenon, resulting in severe thrombocytopenia and bleeding following transfusion of any blood product containing platelet membrane antigens. ${ }^{12}$ In pregnant women who have been alloimmunised, there is a risk of Neonatal Alloimmune Thrombocytopenia in the fetus and they need specialist management in a feto-maternal medicine unit due to risks of intracranial haemorrhage. It is important to limit the risk of alloimmunisation by limiting exposure and using HLA-matched leucodepleted platelet concentrates for transfusion, although random platelets may be needed in an emergency. ${ }^{8,12}$

\section{Conclusion}

This case of HPS was identified and managed during pregnancy with multidisciplinary specialist input. A detailed history helped to guide further investigation and thus establish this rare diagnosis. A comprehensive labour plan prepared the patient for the risk of bleeding at delivery and for the immediate postnatal period although she did well and recovered quickly. Further specialist care has been put in place to monitor the patient for any complications of HPS, or indeed, for any future pregnancies.

\section{Declaration of Conflicting Interests}

The author(s) declared no potential conflicts of interest with respect to the research, authorship, and/or publication of this article.

\section{Funding}

The author(s) received no financial support for the research, authorship, and/or publication of this article.

\section{Ethical approval}

$\mathrm{N} / \mathrm{A}$

\section{Guarantor}

LY

\section{Contributorship}

All authors contributed equally to the drafting of the case report and have provided final approval of the version to be published.

\section{References}

1. Gahl WA and Huizing M. Hermansky Pudlak syndrome. GeneReviews, $\quad$ http://www.ncbi.nlm.nih.gov/books/NBK1287 (2000, accessed October 2015).

2. Hermansky F and Pudlak P. Albinism associated with hemorrhagic diathesis and unusual pigmented reticular cells in the bone marrow: report of two cases with histochemical studies. Blood 1959; 14: $162-169$.

3. Verloop MC, Von Wieringen A, Vuylsteke J, et al. Albinism, heamorrhagic diathesis and pigmented reticuloendothelial cells. Med Klin 1964; 59: 408-412.

4. Witkop CJ Jr. Personal communication. Minneapolis, MN, February 1986. 
5. Wildenberg SC, Oetting WS, Almodovar C, et al. A gene causing Hermansky-Pudlak syndrome in a Puerto Rican population maps to chromosome 10q2. Am J Hum Genet 1995; 57: 755-765.

6. King RA, Hearing VJ, Creel DJ, et al. Albinism. In: Scriver CR, Beaudet AL, Valle DL, et al. (eds) The metabolic and molecular bases of inherited disease. Vol 4, 8th ed. New York: McGraw-Hill, 2001, pp.5587-5627.

7. Hermansky Pudliak Syndrome. Genetics home reference, http://ghr. nlm.nih.gov/condition/hermansky-pudlak-syndrome (accessed 18 October 2015).

8. Sandrock M and Zieger B. Current strategies in diagnosis of inherited storage pool defects. Transfus Med Hemother 2010; 37: 248-258.

9. Schinella RA, Greco MA, Cobert BL, et al. Hermansky-Pudlak syndrome with granulomatous colitis. Ann Intern Med 1980; 92: 20-23.
10. Shimizu K, Matsumoto T, Miura G, et al. Hermansky-Pudlak syndrome with diffuse pulmonary fibrosis: radiologic-pathologic correlation. J Comput Assist Tomogr 1998; 22: 249-251.

11. Wijermans PW and van Dorp DB. Hermansky-Pudlak syndrome: correction of bleeding time by 1-desamino-8D-arginine vasopressin. Am J Hemat 1989; 30: 154-157.

12. D'Andrea G, Chetta $M$ and Margaglione M. Inherited platelet disorders: thrombocytopenias and thrombocytopathies. Blood Transf 2009; 7: 278-292.

13. Kirchmaier CM and Pillitteri D. Diagnosis and management of inherited platelet disorders. Transf Med Hemother 2010; 37: 237-246. 